INPLASY

PROTOCOL

To cite: Zhou et al. A metaanalysis of prevalence and risk factors of Internet pornography addiction among adolescents. Inplasy protocol 202210013. doi:

10.37766/inplasy2022.1.0013

Received: 03 January 2022

Published: 03 January 2022

Corresponding author:

Ruoyu Zhou

378518967@qq.com

Author Affiliation:

University of Tsukuba.

Support: None.

Review Stage at time of this submission: Piloting of the study selection process.

Conflicts of interest:

None declared.

\section{A meta-analysis of prevalence and risk factors of Internet pornography addiction among adolescents}

Zhou, R'; Yang, W2; Wu, M3; Wang, Y4; Wang, L5.

Review question / Objective: To provide an overview of prevalence and risk factor for Internet pornography addiction in adolescents according to meta-analyses.

Condition being studied: Internet pornography addiction: A psychopathic state of being addicted to adult-talking chat rooms and online pornographic literature and videos. Research into the area of addictive sexual behaviors on the Internet began with an inquiry into the various constructs surrounding compulsive sexual behavior.

Information sources: For literature on mindfulness practice for adolescent emotional disorders published before December, 20th, 2021, search databases will include Google Scholar, EMBASE, Web of Science, PubMed, the CNKI, the Chinese Science and Technology Periodical Database, VIP, Wanfang, and Cochrane Library.

INPLASY registration number: This protocol was registered with the International Platform of Registered Systematic Review and Meta-Analysis Protocols (INPLASY) on 03 January 2022 and was last updated on 03 January 2022 (registration number INPLASY202210013).

\section{INTRODUCTION}

Review question / Objective: To provide an overview of prevalence and risk factor for Internet pornography addiction in adolescents according to meta-analyses.
Condition being studied: Internet pornography addiction: A psychopathic state of being addicted to adult-talking chat rooms and online pornographic literature and videos. Research into the area of addictive sexual behaviors on the 
Internet began with an inquiry into the various constructs surrounding compulsive sexual behavior.

\section{METHODS}

Search strategy: For literature on mindfulness practice for adolescent emotional disorders published before December , 20th, 2021, search databases will include Google Scholar, EMBASE, Web of Science, PubMed, the CNKI, the Chinese Science and Technology Periodical Database, VIP, Wanfang, and Cochrane Library.to identify systematic reviews or metaanalyses. The following search terms and their derivatives were used in multiple combinations: "Compulsive sex", cybersex, hypersexual, "hypersexual disorder", imaging, "impulsive sex", "out of control sex", "problem sex", sex, "sex addict", "sexually explicit material", "Internet Pornography Addiction"and"Internet porn addiction".

Participant or population: (1) Adolescents (18-35 years old) with a diagnosis of mood disorder. (2) No limitation on gender or race. (3) No organic brain injury or other neuropsychiatric disorders.

Intervention: Not applicable.

Comparator: Not applicable.

Study designs to be included: Metaanalyses pooling observational (crosssectional, case control, cohort) studies.

Eligibility criteria: We will include studies the met the following criteria: (1) metaanalyses; (2)Adolescents (>20 years old); (3) The studies reported the prevalence or/and risk factors for Internet pornography addiction.

Information sources: For literature on mindfulness practice for adolescent emotional disorders published before December , 20th, 2021, search databases will include Google Scholar, EMBASE, Web of Science, PubMed, the CNKI, the Chinese Science and Technology Periodical
Database, VIP, Wanfang, and Cochrane Library.

Main outcome(s): Prevalence of Internet Pornography Addiction, and risk factors for Internet Pornography Addiction.

Additional outcome(s): None.

Quality assessment / Risk of bias analysis: Quality of included meta-analyses and systematic reviews will be assessed by Stata 16. Two researchers will evaluate the quality of studies, and any discrepancies will be resolved by a third author.

Strategy of data synthesis: Stata $\mathbf{1 6 . 0}$ software was used, Odds rations (OR) and their $95 \% \mathrm{Cl}$ were extracted when reported or calculated from available data in the paper,We will calculate the summary effect size, $95 \% \mathrm{Cl}$, and $\mathrm{p}$ value of eligible metaanalyses with individual study estimates extracted from each meta-analysis.

Subgroup analysis: When the heterogeneity was large, we performed subgroup analysis. According to the characteristics of the included articles, .we will conduct a subgroup analysis to determine the difference between different gender, education background etc.

Sensitivity analysis: Sensitivity analysis will be performed by excluding tests one by one and observing whether there is a significant change in the synthesis results. If $I^{\wedge} 2 \geq 50 \%$ for the primary outcome, further analysis will be performed of each risk factor.

Language: English.

Country(ies) involved: Japan.

Keywords: Internet Pornography Addiction, prevalence, risk factors, umbrella review.

Contributions of each author:

Author 1 - Ruoyu Zhou.

Author 2 - Wenjie Yang.

Author 3 - Ming Wu.

Author 4 - Yu Wang.

Author 5 - Liqiong Wang. 